\title{
Vesical Gossypiboma with Urethral Migration Causing Acute Urinary Retention: A Case Report and Review of the Literature
}

\section{Kaleab Habtemichael Gebreselassie}

St. Paul's Hospital Millennium Medical College

Ferid Ousman Mummed ( $\nabla$ sarson.fo@gmail.com )

St. Paul's Hospital Millennium Medical College

Fitsum Gebreegziabher Gebrehiwot

St. Paul's Hospital Millennium Medical College

Mubarek Bargicho Adem

St. Paul's Hospital Millennium Medical College

\section{Ramzi Yessuf Adem}

St. Paul's Hospital Millennium Medical College

Feysel Hassen Issack

St. Paul's Hospital Millennium Medical College

\section{Case Report}

Keywords: Gossypiboma, surgical sponge, acute urinary retention, case report

Posted Date: January 6th, 2022

DOI: https://doi.org/10.21203/rs.3.rs-1223212/v2

License: (9) (i) This work is licensed under a Creative Commons Attribution 4.0 International License. Read Full License 


\section{Abstract \\ Introduction:}

Vesical gossypiboma is a retained surgical sponge in the urinary bladder following abdominal or pelvic surgery. It is probably under-reported due to medico-legal issues. It usually poses a diagnostic challenge to clinicians. A high index of suspicion is essential to make a timely diagnosis.

\section{Case Presentation:}

We present a case of vesical gossypiboma in a 64-year-old male patient who presented with a history of persistent lower urinary tract symptoms (LUTS) and acute urinary retention following trans-vesical prostatectomy. The urinary retention was relieved by a suprapubic cystostomy. On cystoscopic examination, a large whitish spongiform structure in the urethra and bladder was revealed. A gentle attempt of cystoscopic removal was failed. At exploration, a retained surgical sponge was revealed and removed from the bladder. Postoperatively, the patient reported improvement of symptoms and was passing urine normally.

\section{Discussion}

Vesical gossypiboma is relatively rare as very few isolated cases have been reported, and its migration through the urethra is even rarer. Patients with intravesical gossypiboma may manifest with either acute or chronic symptoms due to complications. Gossypiboma is an avoidable condition, and its occurrence can be prevented by thorough exploration of the surgical site and count of surgical materials at the end of the procedure.

\section{Conclusion}

Vesical gossypiboma should be considered as a differential diagnosis in patients with persistent LUTS following open bladder or prostate surgery. Open surgical removal is one of the feasible treatment options. But prevention is a preferable solution.

\section{Introduction}

Gossypiboma is a retained surgical sponge (RSS) in the body after a surgical procedure. Although intraabdominal gossypiboma is the most common, it has been reported to occur in all surgical disciplines. The incidence of gossypiboma is under-reported, probably due to medico-legal issues and misdiagnosis. There have been few reports of vesical gossypiboma in medical literature and even fewer regarding migration through the urethra [1-3]. 
Patients with intravesical gossypiboma manifest with either acute or chronic urinary symptoms. The most common presentation is lower urinary tract symptoms (LUTS). They may also result in recurrent urinary tract infection (UTI), discharge per urethra, hematuria, and acute urinary retention (AUR) $[4,5]$.

Gossypiboma usually poses a diagnostic challenge to the clinician with significant patient morbidity, probably due to its delayed non-specific presentation and inconclusive imaging findings. The treatment of intravesical gossypiboma has to be individualized depending on the size of RSS and its nature, adhesion, and age of the patient $[3,6]$. Herein, we report a case of vesical gossypiboma with urethral migration after open prostatectomy resulting in AUR. This case report has been reported in line with the SCARE Criteria [7].

\section{Presentation Of Case}

A 64-year-old male patient was referred to our hospital with a history of not passing urine per urethra for two weeks. The patient had developed AUR which was relieved by open suprapubic cystostomy after failed transurethral catheterization. The patient had undergone a trans-vesical prostatectomy for benign prostatic hyperplasia before three months at another hospital.

Postoperatively, the patient had been experiencing persistent irritative and obstructive LUTS. The patient had received oral antibiotics for the recurrent UTI from the local hospital. The abdominal examination revealed an infraumbilical surgical scar with a suprapubic catheter in situ.

Complete blood count, renal function test, and urinalysis were normal and the urine culture revealed no growth. Abdominopelvic ultrasonography was unremarkable. Cystourethroscopy examination was performed with the impression of bladder neck stenosis, and a large whitish spongiform structure impacted in the urethra was revealed [Figure 1].

A gentle attempt to remove the retained sponge using endoscopic forceps was failed. Antegrade cystoscopic evaluation under spinal anesthesia showed the same spongiform structure in the bladder with distal migration through the bladder neck. Again, a gentle attempt of cystoscopic removal was failed, resulting in partially protruded surgical gauze through the suprapubic wound [Figure 2].

Then, the patient was explored via Pfannenstiel incision. On bladder exploration, a retained surgical sponge adhering to the bladder was revealed and removed successfully [Figure 3]. The patient was discharged on the next day after making an uneventful recovery. A transurethral catheter was removed on the $7^{\text {th }}$ postoperative day. The patient reported improvement of his symptoms and was passing urine normally during his follow-up visits.

\section{Discussion}

The term "Gossypiboma" is derived from a combination of the Latin word "Gossypium" (cotton) and Swahili word "Boma" (place of concealment). Although it has been reported to occur in all surgical 
disciplines, the most common location is the abdominal cavity, followed by the pelvis. Gossypiboma is rarely seen in clinical practice due to medico-legal issues. It is estimated to occur in 1/100 - 3000 cases for all surgical procedures. Vesical gossypiboma is relatively rare, and its migration through the urethra is even rarer $[2,3,8]$. In our case, the patient had intravesical retained gauze with urethral migration causing AUR.

Risk factors for RSS include unplanned surgeries of longer duration, operations entailing multiple procedures, emergency surgeries, more than one surgical team, high intraoperative blood loss, failure of a material count or incorrect counts, and obesity $[3,4,9]$. Since our patient was operated on at some other hospital, we couldn't identify the possible risk factors.

Vesical gossypiboma can manifest at any time, ranging from the immediate to several decades after the procedure. The most common presentations include LUTS and recurrent UTI, as in the index case $[3,5]$. The detection of gossypiboma is clinically and radiologically challenging. Ultrasonography, CT (computed tomography) scan, and MRI (magnetic resonant imaging) are used in diagnosis. Cystoscopy has good diagnostic and therapeutic advantages. A high index of suspicion is very important to make a timely diagnosis [2-4].

Once the RSS is diagnosed, it should be removed. The methods of removal depend on the size and its fragility and adhesion. Open exploration is commonly performed, but endoscopic or percutaneous removal can also be performed in selected cases. It is an avoidable condition and prevention is the preferable solution; through a thorough exploration of the surgical site and meticulous count of surgical materials at the end of each procedure $[2,3,8]$. In our case, open bladder exploration was done after a failed trial of cystoscopic removal.

\section{Conclusion}

Intravesical gossypiboma should be considered as a differential diagnosis in patients with persistent LUTS following open bladder or prostate surgery. An open surgical removal is an alternative option of treatment. Gossypiboma is an avoidable condition, and prevention is the preferable solution.

\section{Declarations}

\section{Funding}

No sources of funding

\section{Ethical approval}

No ethical approval was required.

\section{Consent}


Written informed consent was obtained from the patient for the publication of this case report and accompanying images. A copy of the written consent is available for review by the Editor-in-Chief of this journal on request.

\section{Research registration}

N/A

\section{Acknowledgments}

N/A

\section{Declaration of competing interest}

Nothing to declare.

\section{Abbreviations}

AUR; acute urinary retention; CT, computed tomography; LUTS, lower urinary tract symptoms; MRI, magnetic resonance imaging; RSS, retained surgical sponge; UTI, urinary tract infection.

\section{References}

1. Mungadi, I.A., N.A. Attahiru, and S.A. Saidu, Vesical gossypiboma mimicking calculus: a report of two cases. African J. Urol, 2007. 13(2): p. 136-138.

2. Kansakar, R. and B.K. Hamal, Cystoscopic removal of an intravesical gossypiboma mimicking a bladder mass: a case report. J Med Case Rep, 2011. 5(1): p. 579. https://doi.org/10.1186/1752-1947-5579

3. Kori, R., L. Bains, and S.K. Jain, Intravesical gossypiboma: our experience and the need for stringent checklist and training! BMJ Case Reports, 2019. 12(2): p. e227278. https://doi.org/10.1136/bcr-2018227278

4. Siddiqui, Z. and M. Naeem, Gossypiboma bladder: the need to count sponges. J Pak Med Stud, 2011. 1: p. 19.

5. Kumar, B., et al., Gossypiboma mimicking posterior urethral stricture. International Journal of Surgery Case Reports, 2013. 4(4): p. 425-428. https://doi.org/10.1016/j.ijscr.2013.01.020

6. Javanmard, B., et al., Retained Surgical Gauze Presenting with Gross Hematuria: A Case Report. Urol J, 2017. 14(5): p. 5027-5029. https://doi.org/10.22037/uj.v14i5.3735 
7. Agha, R.A., et al., The SCARE 2020 Guideline: Updating Consensus Surgical CAse REport (SCARE) Guidelines. International Journal of Surgery, 2020. 84: p. 226-

230. https://doi.org/10.1016/j.ijsu.2020.10.034

8. Singh, P., S. Panaiyadiyan, and B. Nayak, Pelvic gossypiboma with spontaneous intravesical erosion. BMJ Case Rep, 2017. 2017: p. bcr-2016-219173. https://doi.org/10.1136/bcr-2016-219173

9. Joshi, N., R. Kumar, and V. Yadav, Gossypiboma in the urinary bladder: Time to make new surgical guidelines. Medical Journal of Dr. DY Patil University, 2014. 7(4): p. 489. https://doi.org/10.4103/09752870.135280

\section{Figures}

\section{Figure 1}

Cystourethroscopic view showing a large retained spongiform structure in the urethra

\section{Figure 2}

A piece of surgical gauze protruding through suprapubic cystostomy site after trial of antegrade cystoscopic removal.

\section{Figure 3}

Postoperative picture showing a retrieved surgical gauze. 